\title{
Creación de contenidos transmedia en la sociedad hiperconectada. Una etnografía digital con jóvenes universitarios ${ }^{1}$
}

\author{
Creation of transmedia contents in the hyperconnected society. \\ A digital ethnography with university students.
}

\author{
Rocío Anguita Martínez \\ Laura De la Iglesia Atienza \\ Eduardo García Zamora \\ Universidad de Sevilla
}

Recibido: $17 / 01 / 2018$

Aceptado: 02/05/2017

\begin{abstract}
We present an investigation carried out with university students of the field of social education centred on the analysis of the design, creation and broadcasting of transmedia contents. In addition, we are interested in the role that transmedia contents can play in the creation of participatory cultures and social and community development. First, we make a brief introduction about the role that transmedia literacy and convergence culture can play into the formal education institutions. Second, we show the educational design analysed. Third, the study addresses the main elements that shaped the research design, having opted for a work that combined narrative research with digital ethnography intended not only to describe the process followed, but also to identify the possibilities of transmedia projects in the university for the development of soft skills, the management of tacit knowledge and the collective construction of knowledge. Fourthly, the main results derived from the educational development followed in the elaboration and the production of transmedia content by the students are described. And finally, a series of conclusions is also discussed, in which important issues such as the relationship between transmedia literacy, the construction of citizenship and the mediation of learning from creative experimentation
\end{abstract}

KEY WORDS: Transmedia narrative, educommunication, media literacy, media ecology

\section{RESUMEN}

Se presenta un trabajo realizado con jóvenes universitarios centrado en el análisis del diseño, creación y difusión de contenidos transmedia y el papel que éstos pueden jugar para el establecimiento de culturas participativas y el desarrollo social y comunitario. Tras realizar una breve introducción respecto del papel que puede jugar la alfabetización transmedia y la cultura de la convergencia dentro de las instituciones de educación formal, se expone el diseño educativo que ha sido objeto de investigación. Posteriormente, el estudio aborda los principales elementos que conformaron el diseño de investigación, habiéndonos decantado por un trabajo que combinando la investigación narrativa con la etnografía digital pretendía no sólo realizar una descripción del proceso seguido, sino, también, identificar las posibilidades de los proyectos transmedia en la universidad para el desarrollo de habilidades blandas, la gestión de saberes tácitos y la construcción colectiva de conocimiento. En un tercer momento se describen los principales resultados derivados del desarrollo educativo seguido en la elaboración y producción del contenido transmedia por el alumnado. Finalmente, se enumeran una serie de conclusiones en las que se discute además respecto de asuntos tan importantes como la relación entre la alfabetización transmedia, la construcción de ciudadanía y las mediaciones del aprendizaje desde la experimentación creativa.

PALABRAS CLAVE: Narrativas transmedia, educomunicación, alfabetización mediática, ecología de los medios.

\footnotetext{
${ }^{1}$ Este texto resultado del proyecto de investigación "Ecologías del aprendizaje en contextos múltiples: análisis de proyectos de educación expandida y conformación de ciudadanía” (EDU2014-51961-P). Ministerio de Economía y Competitividad. Proyectos de I+D. Convocatoria 2014, Modalidad 1: Programa Estatal de Fomento de la Investigación Científica y Técnica de Excelencia, Subprograma Estatal de Generación del Conocimiento
}

Dirección de correspondencia:

Rocío Anguita Martínez, Universidad de Valladolid, Dpto. de Pedagogía. Facultad de Educación y Trabajo Social.. E-mail: rocioan@pdg.uva.es 


\section{Introducción}

En la actualidad, las culturas participativas tienden a ser vistas cada vez más como un proceso centrado en los entornos tecnológicos (Van Dijck, 2013) donde más que hablar de personas que son productores y consumidores mediáticos, podríamos verlas como participantes que interaccionan para construir su propia mitología personal con los recursos extraídos del flujo mediático y que confieren sentido a su vida cotidiana (Jenkins, Ito \&Boyd, 2015).

La convergencia de medios no tiene lugar mediante aparatos tecnológicos, sino que se produce en el cerebro de los consumidores individuales y mediante sus interacciones sociales con otros consumidores (Jenkins, 2006). Esta convergencia representa un enorme cambio cultural ya que anima a los consumidores a buscar nueva información y establecer conexiones entre contenidos mediáticos dispersos. Este proceso genera una inteligencia colectiva que puede verse como una nueva alterativa de poder mediático.

Uno de los fenómenos que emergen de estas culturas participativas son las narrativas transmedia (Scolari, 2016). En el nivel más básico, los relatos transmedia son historias contadas a través de múltiples medios de comunicación. Las historias más importantes tienden a fluir a través de múltiples plataformas y medios de comunicación (Wängqvist, M. \& Frisén, A. 2016). Desde la perspectiva de los consumidores, las prácticas transmedia promueven la multialfabetización, o sea, la capacidad de interpretar de manera integral los discursos procedentes de diferentes medios y lenguajes. Por otra parte, no podemos perder de vista el papel que juegan los medios de comunicación en su configuración cognitiva social y afectiva (Jenkins, Ford \& Green, 2013), los usos y apropiaciones que hacen de las tecnologías y artefactos digitales (Bar, Weber \& Pisani, 2016), el rol que juegan los social media como instrumentos del empoderamiento social y ciudadano al crear subculturas y formatos colaborativos de generación, distribución y recepción de la cultura mediática (Buckingham \& Kehily, 2014).

Desde el punto de vista educativo el uso de las tecnologías digitales ha supuesto una oportunidad para el ejercicio de nuevas formas de interacción social que, en la actualidad, están transformando el funcionamiento y rol de las instituciones de aprendizaje formal, sobre todo las escuelas y la universidad (Malone, T. W., Bernstein, M. S., 2015). Uno de los retos más importantes que debemos afrontar es el de que todas esas experiencias en las que se desarrollan, extienden y difunden nuevas formas de producción, comunicación y adquisición de conocimiento generadas en ámbitos de naturaleza y procedencia diversa tengan una traducción a nivel educativo, y se transformen en procesos de aprendizaje integrales (Ito, 2010). En este marco, los medios digitales abren la puerta a un nuevo paradigma educativo en el que el aprendizaje pueda tener lugar "en cualquier momento y en cualquier lugar", una dinámica cultural que ha sido descrita en la literatura como ubicua (Cope \& Kalantzis, 2009) y que nos recuerda que la vida diaria se convierte en espacio para nuevas pedagogías y nuevas prácticas de aprendizaje.

En este sentido, debemos tener en cuenta las posibilidades que ofrece el diseño, creación y difusión de contenidos transmedia en tanto proceso de mediación tecnológica y social (Rao, Jongerden, Lemmens, \& Ruivenkamp, 2015) respecto de la visibilización, reconocimiento y configuración de una serie de habilidades blandas y saberes tácitos que los y las estudiantes pueden traer consigo (Dede, 2010; Davies, Fidler \& Gorbis, 2011), y que han sido caracterizados por la literatura bajo el marco general de aprendizajes invisibles en una sociedad knomádica (Moravec, 2013; Cobo, 2016).

\section{Metodología de la investigación}

El objetivo de la investigación realizada ha sido profundizar en el análisis del diseño y creación de narrativas transmedia elaboradas por jóvenes universitarios en el marco de culturas participativas en red, combinando la creación de contenidos multimedia con propuestas educativas orientadas al desarrollo social y comunitario.

Se trata de comprender cómo estos jóvenes están adquiriendo competencias transmedia e incorporando estos procesos al ámbito educativo (Vélez, 2017), donde aprender es un efecto colateral 
de la producción creativa y de la colaboración comunitaria, lo que se denomina "connected learning" o aprendizaje en conexión (Ito, 2010).

Los objetivos específicos que se querían alcanzar con la investigación eran:

1. Identificar en el universo mediático de la ficción o de la comunicación social que analizan y elaboran los y las jóvenes, es decir, cuáles son las características e indicadores principales en el diseño y creación de narrativas transmedia.

2. Indagar acerca del papel que juega el diseño educativo de propuestas transmedia y los procesos de mediación tecnológica basados en la cultura participativa en red, respecto a la gestión de saberes tácitos del alumnado, el desarrollo de habilidades blandas y la transferencia de aprendizajes al ámbito personal, profesional y social.

3. Explorar las formas de participación y procesos de construcción colectiva de conocimiento en la creación de narrativas transmedia de los jóvenes universitarios y el impacto que tienen en la configuración de subjetividades ciudadanas.

El estudio realizado parte de una investigación de corte narrativo (Chase, 2011; Denzin\& Lincoln, 2011; Rivas Flores, 2014), ya que el foco de atención se centró en la exploración de las producciones narrativas transmedia a través de las que los y las participantes conforman modos de actuar y configurar sentidos en el entorno hiperconectado

En el plano metodológico se realizó una etnografía digital (Hine, 2004; Beaulieu, 2004) ya que nos permite examinar las relaciones que mantienen las y los jóvenes con las tecnologías y los medios de comunicación y cómo estos últimos median en la configuración de subjetividades contemporáneas y culturas participativas. La perspectiva etnográfica que usamos tiene que ver con los desafíos que propone internet y su uso, tanto como herramienta de investigación como objeto de conocimiento (Ardévol, et. al, 2008) al tener en cuenta una serie de elementos novedosos que añade internet a la investigación tales como (Hine, 2004) el ser "multi-local" permite considerar la comunicación analógica y digital como dos caras de una misma vivencia y, por ello, ser fluida, móvil, cambiante y dinámica, para permitir entender las relaciones entre lo virtual y lo presencial. Por ello mismo también obliga a los y las investigadores a entender y vincularse con la tecnología que se está analizando y a empatizar con las formas de relación que propician estas tecnologías.

En el caso concreto que presentamos, la vivencia virtual y real se han dado la mano constantemente, ya que todas las producciones realizadas por los y las jóvenes, tanto en formato audiovisual como en formato textual, se han realizado en formato digital. Además, ellos y ellas han expuesto su narrativa transmedia analizando sus expansiones en los espacios virtuales: redes sociales, webs, blogs, chats,... todo ello mezclado con sus apreciaciones y vivencias entre la vida analógica y presencial

El proceso de investigación se llevó a cabo durante el curso académico 2015/16 en el marco de dos asignaturas pertenecientes al Grado de Educación Social en la Universidad de Valladolid (Castilla y León-España): TIC’s Aplicadas a la Educación (primer curso) y Medios de Comunicación Social (cuarto curso), en la Facultad de Educación y Trabajo Social de Valladolid y la Facultad de Educación de Palencia, respectivamente.

El estudio incluye el trabajo y análisis tanto de los aspectos vinculados a la realidad analógica y presencial del aula como a los elementos de la docencia y la realidad virtual/digital de los y las participantes. El volumen ingente de material recogido ha obligado a realizar una selección para el análisis y posterior uso en la investigación. Por ello, se seleccionaron 10 producciones de entre todas las narrativas transmedia realizadas en clase.

Los instrumentos y fuentes de datos de investigación utilizados para realizar la investigación responden a las complejidades propias de la etnografía digital:

1. Narrativas transmedia: son las producciones transmedia elegidas por los diferentes grupos para su análisis, tanto en el ámbito de la ficción (p.e. literatura, cine, música, videojuegos, etc.) como en el de la realidad social (p.e. periodismo y documentación social)

2. Expansiones narrativas transmedia: son las producciones audiovisuales elaboradas por los y las jóvenes y que expanden la narrativa transmedia elegida inicialmente, orientadas a un ámbito de la intervención sociocomunitaria. 
3. Observaciones de aula: En las dos aulas donde se ha desarrollado la investigación ha habido un observador externo, que ha realizado observaciones sobre la dinámica de trabajo en el aula de los y las jóvenes en el proceso de realización de las narrativas transmedia.

4. Comentarios y recomendaciones realizadas en el trabajo en grupo: se han recogido todos los comentarios y textos realizados a los trabajos realizado en grupo por los y las jóvenes.

5. Grabaciones en video: todos los procesos del diseño, creación, presentación y puestas en común en las clases de los trabajos de los estudiantes han sido grabados en video.

Para la codificación y categorización de datos hemos seguido tres procedimientos sistemáticos y exhaustivos, con los que se buscaba generar comprensiones sobre el objeto de estudio gradualmente:

1. Procesos de codificación y categorización: son procesos sistemáticos y exhaustivos en los que se busca generar comprensiones sobre el objeto de estudio, para lo cual se utilizó un procedimiento de codificación posterior a partir de los datos (Strauss \&Corbin, 1990; Trinidad, Carrera \& Soriano, 2006).

2. Mapeo conceptual de las representaciones gráficas y audiovisuales: procediendo a la representación de conocimientos de forma visual, lo cual nos ha permitido enlazar con conceptos afines a la hora de proceder a la interpretación de datos (Simons, 2009).

3. Análisis discursivo de las narrativas: al centrarnos no sólo en la descripción de lo que las narrativas dicen y su estructura formal, sino también el significado de la experiencia con relación al proceso de selección, producción y presentación de las expansiones narrativas seleccionadas en el estudio (Gibbs, 2012).

\section{Diseñando contenidos transmedia en el ámbito de la formación en el Grado de Educación Social: la propuesta educativa}

El proceso educativo parte de considerar el diseño, producción, presentación y análisis de las narrativas transmedia en tanto actividad sociocultural en la que se entrecruzan tres planos (Rogoff, 1997), el de la apropiación participativa, el de la participación guiada y el del aprendizaje.

Nuestra propuesta se centra en el plano de la participación guiada y cómo implementar procesos educativos que medien los aprendizajes de las y los estudiantes de forma colaborativa. En esta propuesta se dan tres procesos de apoyo mutuo en la configuración de aprendizajes:

1. Por un lado, implementando una infraestructura tecno-institucional en la que se consideran diferentes escenarios de trabajo donde experimentar distintas formas de comunicación (hipertextual, multimedial, interactiva, reticular) que abren las puertas a la consideración de un modelo de aprendizaje en el que se comparten objetos, se establecen procesos creativos de conocimiento, dinámicas de interacción mediación e hibridación tecnológica y procesos de transformación personal y profesional).

2. Por otro lado, concibiendo la mediación educativa propuesta en las aulas como un proceso en el que se visibilizan, reconocen y gestionan toda una serie de habilidades blandas del alumnado como son el desarrollo de la creatividad y de la innovación, habilidades de colaboración y de aprendizaje contextual, la posibilidad de generar proyectos de dirección autónoma y en el que se posibilitan habilidades para la comunicación, la selección de información y el pensamiento crítico.

3. Finalmente, tratando de recuperar esos aprendizajes prácticos que han realizado nuestros estudiantes en la sociedad hiperconectada, para lo cual el aula se convierte en un espacio en el que mediar en la conversión de todos aquellos saberes tácitos que las y los jóvenes universitarios aprenden en y a través de sus interacciones digitales.

El diseño educativo que propusimos para realizar esta investigación se presenta en seis fases o momentos, tal y como se puede ver en la Figura 1. 


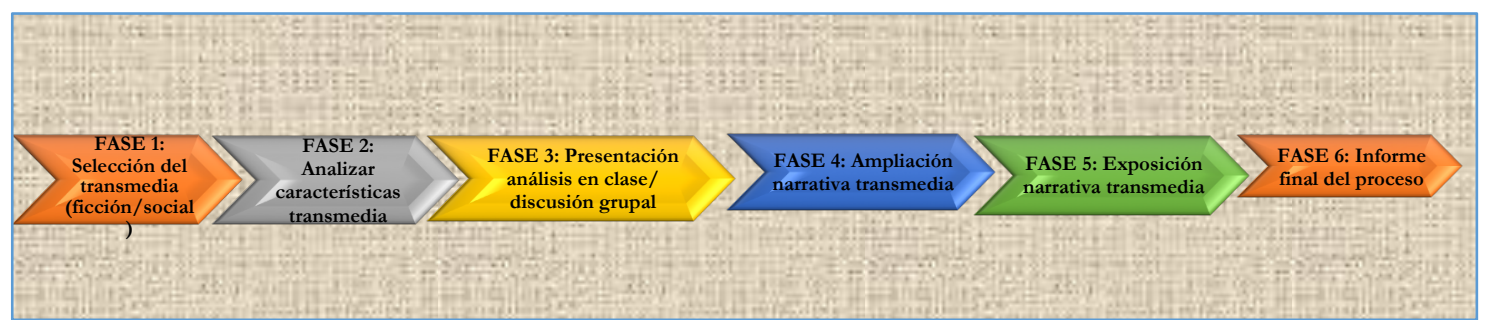

Figura 1. Fases en el diseño, creación y difusión de Narrativas Transmedia

- En la primera y segunda fase los y las estudiantes comparten sus propias experiencias mediáticas de forma colectiva por grupos de trabajo (socialización) a través de la selección y análisis de una producción transmedia;

- En la tercera fase se produce un primer proceso de intercambio sobre las narrativas transmedia en tanto producciones digitales en las que se entremezclan los saberes culturales y cívicos de las y los estudiantes (externalización);

- En la cuarta fase, los y las estudiantes han de interrogarse respecto de algunos ejes o ideas-fuerza de la sociedad aumentada y de su propio capital informacional a través de la ampliación de la narrativa que han analizado previamente (combinación);

- En la quinta y sexta fase del proceso, se vuelve análisis colectivo y la reflexión final respecto de la sociedad aumentada incorporados en un documento sobre proceso seguido durante las sesiones (internalización).

Otro proceso que media en el proceso de apropiación tecnobiográfico-mediática por parte de los y las estudiantes son las dos modalidades de participación que se han desarrollado en el aula, con un carácter eminentemente colaborativo-participativo y que nos han permitido mediar en el capital informacional de los jóvenes hiperconectados:

- Por una parte, la opción de convertir el proceso de creación-diseño-presentación-difusión de las narrativas transmedia en una forma de experimentar y jugar con y respecto a lo que ha sido la constitución identitaria de nuestro alumnado en la sociedad aumentada, pero también en la posibilidad de generar compromisos por parte de los jóvenes hiperconectados respecto del acceso, uso, construcción de ciudadanía que hacemos en y con las tecnologías digitales.

- Por otra parte, la propuesta de trabajo se desarrolla sobre la base de una cierta cultura de la propagabilidad de los saberes tácitos del alumnado respecto de la sociedad hiperconectada, y que se concreta con una propuesta de apropiación práctica (intrapersonal) basada en el flujo de ideas, la selección en base a un material disperso, experiencias mediáticas diversificadas, modelos de participación de las narrativas transmedia de "final abierto", la facilidad para la difusión de los trabajos como instrumento de motivación, la colaboración en los roles desarrollados por el alumnado (creador de historias, analista, colaborador, problematizador...).

Por último, esta cultura de trabajo colaborativo en el aula-clase ha evidenciado diferentes implicaciones y formas de participación respecto a la experiencia propuesta. Desde aquellos/as estudiantes que han jugado un papel de "merodeadores" hasta los que han desarrollado formas de participación periférica al grupo o los que se han comprometido con la propuesta más allá del horario de clases.

Debemos resaltar aquí que esta propuesta de trabajo de contenidos transmedia en el aula es original de las y los autores de la investigación y va en consonancia con otros dispositivos de investigación que se han puesto en marcha para indagar acerca de las producciones transmedia de los jóvenes (Scolari, 2018).

\section{Principales resultados del proceso de diseño, creación y difusión de Narrativas Transmedia en los casos estudiados.}


El primer elemento de reflexión debe ser el mensaje central elegido por el alumnado para elaborar su Narrativa Transmedia (en adelante, NT). En la asignatura se plantea un primer paso que es la elección de la narrativa a partir del conjunto de las producidas en el universo de ficción o en los entornos mediáticos vinculados a la realidad. Partimos de que en la propuesta práctica del aula (que ha tenido lugar tras las sesiones teóricas de la presentación de las NT) se ha dado total libertad para elegir el mensaje, la única recomendación (que no obligación) ha sido que fuera un tema en el que todos los miembros del grupo pudieran aportar algo, para que fuera un verdadero trabajo colaborativo donde cada uno hace lo que mejor sabe hacer, como afirmaba Jenkins (2003). No se hizo mención expresa a que fuera un mensaje vinculado a algunos de los ámbitos de la Educación Social, sino que se dejó completa libertad de elección a los grupos.

La única propuesta alternativa que se ofreció fue que eligieran entre temas de ficción o temas más allá de la ficción. Los grupos optaron por temas de ficción en su totalidad salvo en el caso de un Trabajo de Fin de Grado (TFG) que por razones académicas se vinculó al transmedia de tipo social. Tras un debate en los grupos, cada uno eligió como tema para sus narrativas los siguientes géneros (añadimos la codificación de los datos que utilizamos en adelante):

- Un cuento clásico: La Bella Durmiente - BDMPa

- Una obra literaria: El Señor de los Anillos (influidos por versión cinematográfica) - ESAVa

- Un videojuego: Pokemon - POKVa

- Una serie de TV: Breaking Bad - BKIPa

- Un comic: The Walking Dead (aunque inclinados a su versión como serie de TV) - PTZVa

- Evaluación proyectos sociales de Comunicación Transmedia Social: Mujeres en Venta: trata de personas con fines de explotación sexual en Argentina y Aylan Project: Yo no te olvido PST_1Pa/PST_2Pa.

El alumnado con el que trabajamos las narrativas transmedia podemos decir que no se ajustaba al perfil "clásico" del alumnado del grado de Educación Social -la imagen socialmente construida que tenemos de este colectivo- de ser unos jóvenes con preocupaciones socioeducativas relevantes y mostrarlas en sus producciones académicas, salvo en los casos de los grupos que hemos denominado BDMPa con un claro mensaje reivindicativo sobre el tema de género (Fueyo y De Andrés, 2017) y PSTPa con un mensaje sobre problemáticas sociales vistas a través de los mass media. $\mathrm{El}$ resto de grupos y narrativas tienen un mensaje central asociado a la cultura juvenil de las y los participantes que no tiene nada que ver con su perfil académico y que van desde la adaptación de un videojuego a su ciudad (PKMVa), una serie de TV con un guión adaptado a su ocio de consumo como Breaking Bad (BKIPa) y un par de versiones alternativas para un comic/serie TV (PTZVa) y para una obra literaria/trilogía cinematográfica (ESAVa).

Lo cierto es que el tema elegido por cada grupo nos permite mapear la cultura de los jóvenes universitarios, asunto del que ya teníamos información previa gracias al Cuestionario sobre Identidades Mediáticas "Comunicación, tecnologías e identidad" que habíamos estado trabajando con ambos grupos. Videojuegos, series de TV y cine forman parte de su cultura mediática y esa seguramente sea una de las causas de la elección. Que sean todas las elecciones sobradamente conocidas por todos los y las jóvenes nos habla de la uniformidad de la cultura juvenil, cuando no del pensamiento único y de un consumo masivo de los mismos productos.

Llama también la atención que a pesar de la diferencia de edad entre los dos grupos (de media 18 años en Valladolid, 22 en Palencia) las temáticas son muy semejantes, ¿pertenecen a la misma generación? Mucho se ha hablado de este tema con denominaciones más o menos acertadas, desde nativos digitales a millennials, pero más que su edad nos debería interesar su cultura "popular": ¿Cuáles son sus referentes literarios, cinematográficos, de ocio, de los diferentes mass media? ¿Realmente tienen alguno? Claro que sí, pero seguramente no dentro de nuestros “académicos" parámetros. Que G. Bush aparezca en la NT de PTZVa, Matrix en ESAVa o el Universo Disney en BDMPa nos indica que realmente tienen una cultura que se relaciona directamente con el mensaje que desean transmitir con su NT. 
Una vez elegido el tema de las NT cada grupo llevó a cabo un segundo paso que consistía en analizar las características transmedia de su elección incorporando una serie de indicadores (adaptado de Jenkins y sus posts en el blog Confessions of An Aca-Fan) proporcionados por los responsables de las asignaturas a modo de Guía, pero podían expandirse todo lo que considerasen oportuno. Lo que se denomina Identikit (Scolari, 2013): Expansión (Spreadability) vs. Profundidad (Drillability); Continuidad (Continuity) vs. Multipilicidad (Multiplicity); Inmersión (Immersion) vs. Extraibilidad (Extractability); Construcción de mundos (World building); Serialidad (Seriality); Subjetividad (Subjectivity); Realización (Performance). Estos análisis pudieron realizarse en formato visual. Tenemos el ejemplo de dos de ellos en la Imagen 1.
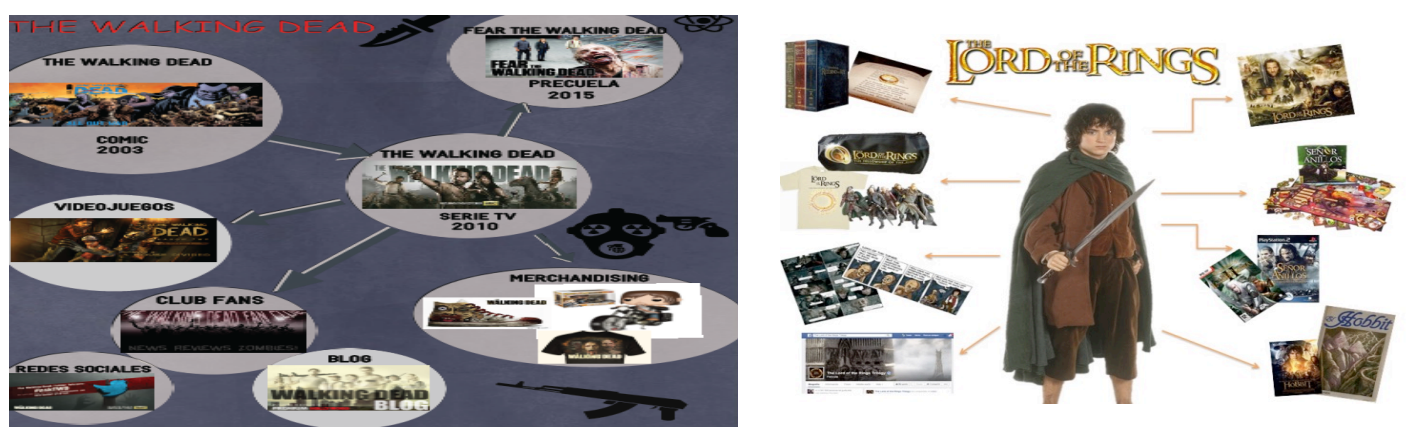

Imagen 1. Análisis de las características transmedia PTZVa, ESAVa

Este análisis en grupo, supervisado en el aula por los profesores responsables de las asignaturas, tuvo como producto final una reflexión escrita elaborada por cada grupo en la que analizaba en profundidad la expansión de su narrativa elegida (Imagen 2).

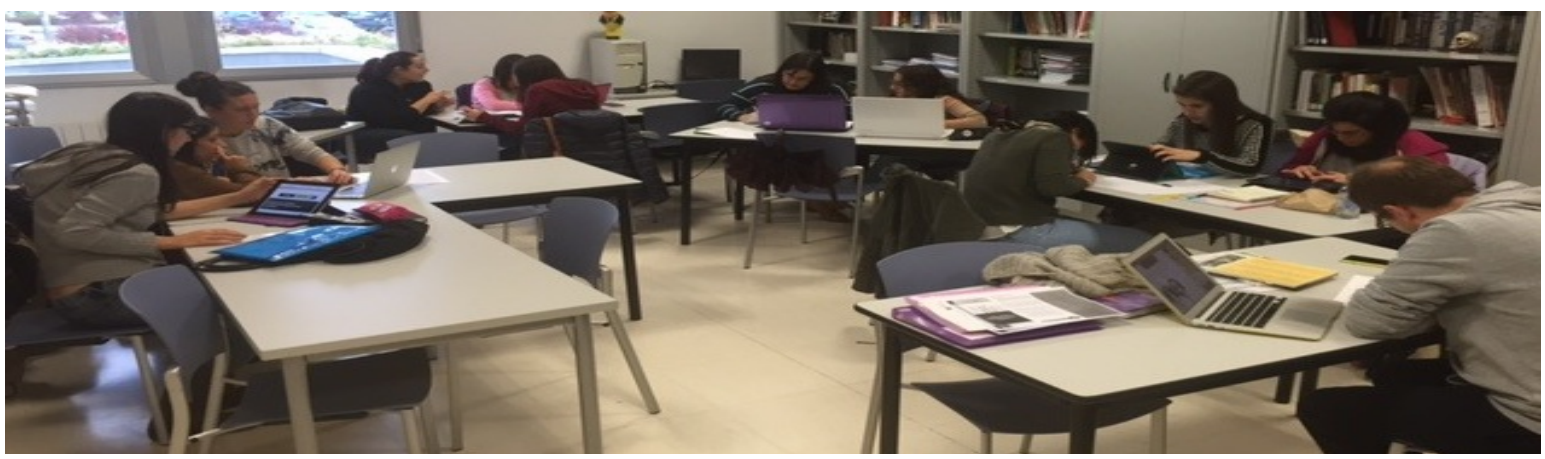

Imagen 2. Alumnado trabajando por grupos en las expansiones $(\mathrm{Pa})$

En un tercer paso, con las exposiciones en grupo de sus análisis en presentaciones elaboradas por el alumnado, se daba paso al co-análisis de las narrativas seleccionadas, tratando de visualizar, conocer y compartir los sentidos y significados, los elementos y las estructuras de cada composición narrativa. Se trataba de que cada grupo tomase nota de todos los comentarios realizados por el resto de grupos de clase, registrando las evidencias del proceso, para en última instancia, redactar un texto descriptivo y comprensivo en el que apareciesen, además del proceso de análisis de cada grupo, las discusiones generadas en el grupo-clase.

A su vez, de acuerdo con el cuarto paso, cada grupo analizaba su propio comportamiento de consumo cultural, analizando e incorporando al informe final algunas dimensiones vinculadas a la identidad y la subjetividad como audiencias que participan en la cultura-red de los mundos transmedia, incorporando algunos análisis que tenían que ver con los modelos participativos y los roles de usuario de consumo transmedia. Se trataba de que cada grupo tomase nota de todos los comentarios, registrando las evidencias del proceso para, en última instancia, redactar un texto 
descriptivo y comprensivo en el que apareciesen, además del proceso de análisis de cada grupo, las discusiones generadas en el grupo-clase.

Con esto llegamos al quinto paso, donde cada grupo ampliaba la narrativa transmedia seleccionada y analizada elaborando una producción propia repensando el mensaje central, elaborando una línea básica narrativa, eligiendo el género al que iba a pertenecer su producción, decidiendo el punto de vista de la historia que se iba a narrar, qué si iba a contar, a qué audiencia iba a estar dirigido. En definitiva, decidiendo los contenidos de su narrativa transmedia como paso previo a su elaboración.

Tabla 1

Producciones finales de las NT expandidas por el alumnado y sus enlaces

El Señor de los anillos (ESAVa): https://www.youtube.com/watch?v=3WnCvr9qLyQ

Potaje Z (PTZVa):

https://goanimate.com/videos/0qCygOAr hDw?utm source=linkshare\&utm medium=linkshare

\&utm campaign=usercontent

Pokemon llega a la Tierra $(\mathrm{POKV}$ ):

https://goanimate.com/videos/0qCygOAr hDw?utm source=linkshare\&utm medium=linkshare \&utm campaign $=$ usercontent

Breaking Ice (BKIPa): https://www.youtube.com/watch?v=RxFCb2C1ls4

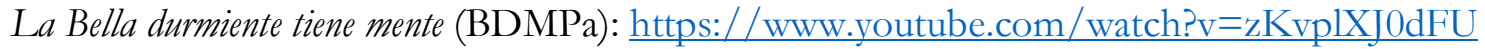

Proyectos sociales transmedia: la comunicación digital como herramienta para la participación ciudadana:

(PST_1Pa/PST_2Pa): http://uvadoc.uva.es/handle/10324/19349

En la Tabla 1 ofrecemos los enlaces donde se encuentran las producciones finales de expansión elaborada por cada grupo y en las Imágenes 3, 4 y 5 podemos ver el aspecto visual del desarrollo de dichas expansiones por el alumnado. Los formatos de estas expansiones fueron variados. Varios optaron por hacer un video con finales y/o contenidos alternativos como el caso de la expansión de la Bella Durmiente (BCMPa, Imagen 3) y el Señor de los Anillo (ESAVa, Imagen 5). Otros grupos optaron por el cómic en formato digital como el caso de la expansión de Pokemon (POKVa, Imagen 5) y The Wlaking Dead y otros optaron por presentaciones en diferentes formatos digitales más clásicas con Powerpoint.

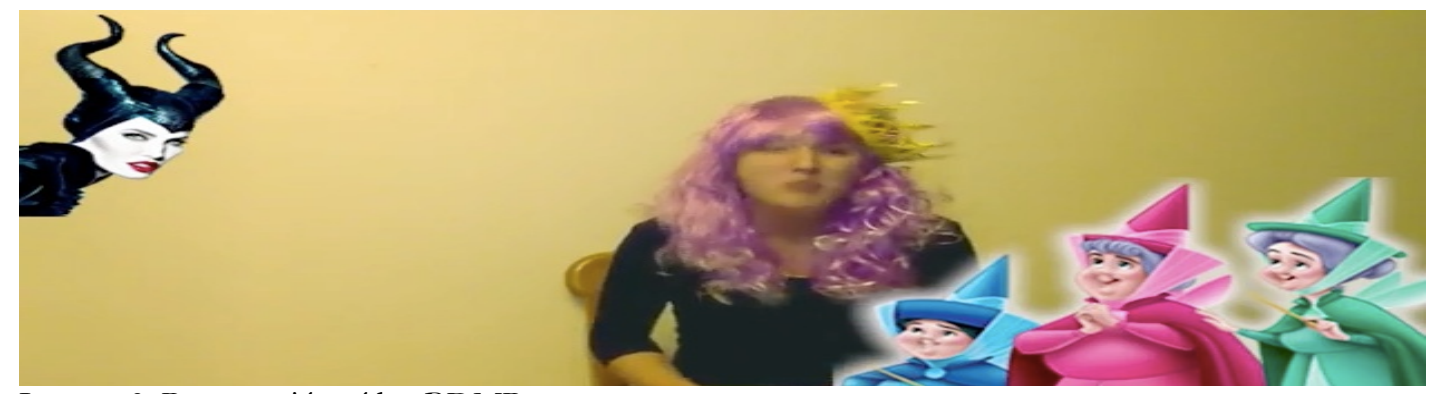

Imagen 3. Presentación vídeo BDMPa 

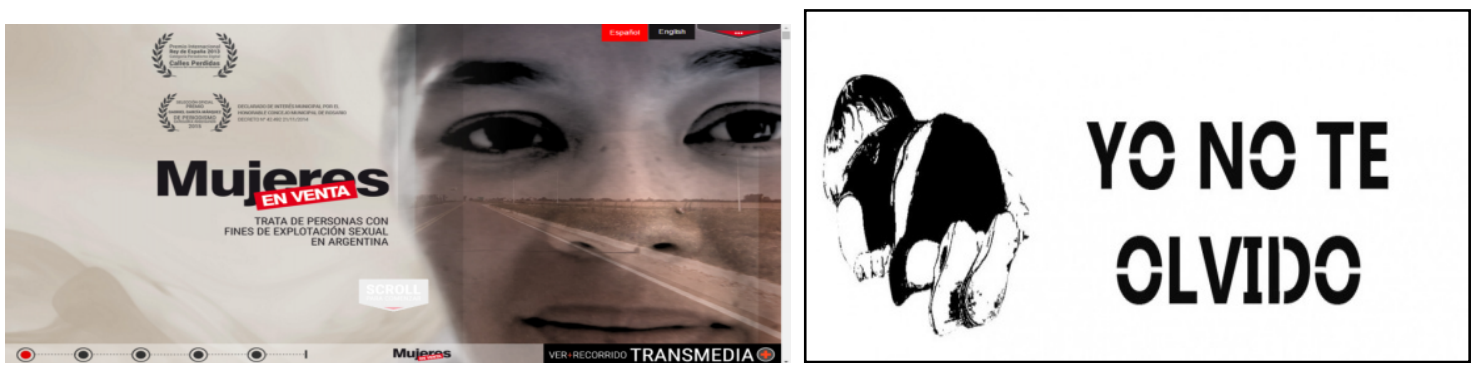

Imagen 4. Proyectos de Comunicación Transmedia PST_1Pa/PST_2Pa

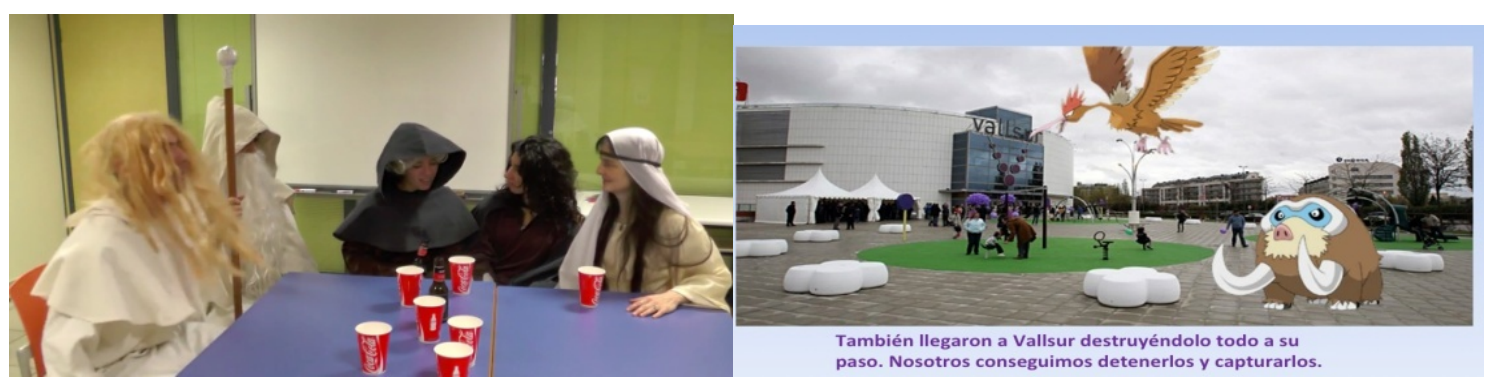

Imagen 5. Presentación Expansión Transmedia, ESAVa y POKVa

Finalmente, en el séptimo paso, cada grupo mostraba al resto de grupos la NT elaborada con un nuevo debate en el aula con aportaciones del resto de grupos, en un intento de socializar las creaciones e imaginarios ofrecidos, pero también como forma de realizar un análisis general respecto de las posibilidades de los universos transmedia.

\section{Discusión de resultados y conclusiones}

Organizaremos esta discusión de resultados y las primeras conclusiones en base a los tres objetivos que nos habíamos propuesto en la investigación.

Respecto al primer objetivo que nos proponíamos, analizar las características del universo mediático de la ficción que elaboran las y los jóvenes, podemos decir que las historias que han sido creadas por los y las estudiantes universitarios nos dicen mucho acerca de cómo el entramado tecnomediático configura imaginarios y representaciones bien diferenciados a nivel afectivo, cultural y político (Schrøder, 2012). Así, podemos contemplar en el alumnado producciones que abordan en forma experimental y creativa los aspectos más lúdicos y hedonistas de su consumo cultural de franquicias del entretenimiento (Pokemon) o de universos narrativos (El Señor de los Anillos) y de la industria televisiva (Breaking Bad, Walking Dead), mientras que otras creaciones orientan el trabajo hacia la crítica feminista de universos clásicos de Disney (La Bella Durmiente) o la denuncia social y el análisis de situaciones relacionadas con los refugiados (Aylan Project).

Ahondando en las características de las narrativas transmedia generadas por el alumnado, hemos comprobado que éstas han sido creadas a través de múltiples medios y lenguajes (cortometraje, dibujo animado, blog, videotube, presentaciones online), por estudiantes que de consumidores/as de los mass media pasan a tener un papel de productores culturales (prosumidores/as) que contraponen ciertas tácticas y estrategias de resistencia frente a los usos hegemónicos y unidireccionales que les son ofrecidos por parte de las creaciones mediáticas originales(p.e. la parodia y el humor desde la crítica social). Además, estas producciones no siguen una estructura lineal sencilla en la que podemos contemplar claramente un guión narrativo del tipo inicio-nudo-desenlace, abriéndose las creaciones de los y las estudiantes a estructuras más polimorfas y desde luego no-lineales (Tubau, 2011), en las que los contenidos claramente responden a un saber generado en la cultura popular de masas pero 
decodificado por los y las estudiantes (Hall, 2001) desde una recepción activa (Simons, 2011; Wood, 2014). En este sentido, nuestra investigación con jóvenes universitarios nos lleva a la necesidad de reconsiderar los formatos y efectos que nutren los diversos universos de la cultura transmedia (Georgi \& Glaser, 2016), sobre todo los procesos de conformación ideológica que hay detrás de buena parte del transmedia de tipo ficción (Hall, 1982; Scolari, Bertetti \& Freeman, 2014; Stein, 2015), pues requiere de una toma de conciencia crítica respecto de los contenido y tramas, intereses comerciales implicados en su desarrollo y audiencias a la que se dirigen (Booth, 2010).

Sobre el segundo objetivo donde indagamos sobre el papel del diseño educativo de proyectos educativos transmedia que hemos puesto en marcha en términos de mediación tecnológica podemos decir que este diseño nos ha permitido configurar una nueva ecología educativa (Cobo \& Moravec, 2011) en el aula universitaria. Los dos agentes del proceso educativo (el profesorado y las y los estudiantes) han podido desarrollar roles diversos durante todo el proceso, tales como consumidores/as culturales, profesionales de la acción social y comunitaria, inician procesos abiertos y participativos de producción de conocimientos.

El caso de estudio que hemos presentado apunta a las posibilidades que ofrece el diseño, creación y difusión de contenidos transmedia en tanto proceso de mediación tecnológica y social (Rao, Jongerden, Lemmens, \& Ruivenkamp, 2015) respecto de la visibilización, reconocimiento y configuración de una serie de habilidades blandas y saberes tácitos que los y las estudiantes traen consigo (Dede, 2010; Davies, Fidler \& Gorbis, 2011), y que han sido caracterizados por la literatura bajo el marco general de aprendizajes invisibles en una sociedad knomádica (Moravec, 2013; Cobo, 2016). En este sentido, en la investigación identificamos claramente con el desarrollo de una serie de habilidades blandas (Livingstone, 2012) que se generan a lo largo de todo el proceso de diseño, análisis, producción y difusión del contenido transmedia y que podemos resumir en las siguientes: capacidad de creación e innovación cultural a partir de una problemática inicial que se plantea con final abierto (expandir el transmedia), capacidad de seleccionar información relacionada con el transmedia elegido, colaboración durante el proceso y aprendizaje contextualizado sobre la base de intereses compartidos vía consumo cultural, autonomía en la dirección dada a los proyectos transmedia generados y la incorporación de una óptica de crítica social (Atkinson, 2014).

La práctica pedagógica vinculada con la creación de contenidos transmedia debe mantener una línea convergente junto a todas aquellas propuestas y experiencias educativas orientadas hacia la construcción dialógica de los saberes y aprendizajes (Barbero, 1987; Thompson, 1991; Garham, 2000; Castells, 2006; Berry, 2015; Allmer, 2015).

Nuestro tercer objetivo de investigación se centraba en explorar las formas de participación y procesos de construcción colectiva de conocimiento en la creación de narrativas transmedia de los jóvenes universitarios y el impacto que éste proceso tiene en la configuración de subjetividades ciudadanas. En la mayoría de los casos se apuesta porque esta generación de contenidos transmedia se conviertan en una oportunidad para generar redes que el ámbito del trabajo comunitario, y en tanto educadores y educadoras, nos lleve a promover ideas basadas en el bien común (Hess \& Ostrom, 2016) y la recuperación de lo público (Soriano, 2016). Las narrativas transmedia realizadas por nuestros estudiantes nos hablan de la posibilidad de combinar lenguajes y medios, construir historias polifónicas donde es posible abordar la historia de forma no lineal, generando receptores activos (prosumidores), lo que es extraordinariamente relevante cuando hablamos del campo de la intervención social y comunitaria y las posibilidades de generar una praxis social transformadora.

Los diseños educativos como el que hemos estudiado nos permiten reintroducir en las aulas universitarias asuntos relacionados con la ciudadanía, el procomún y las prácticas educativas orientas al agenciamiento social (Fleming, 2013; Kalogeras, 2014). Por un lado, porque el establecimiento de redes de trabajo de tipo participativo y deliberativo en torno a la creación de contenidos transmedia recupera una cierta perspectiva demo-diversa (Dahlgren, 2013) en la que reconocemos intereses y saberes, así como la necesidad de conformar en el alumnado un capital social, cultural y político (Gil de Zúñiga, Jung \& Valenzuela, 2012) capaz de luchar contra la exclusión social (Gea, Alaman \& Rodriguez, 2016) que sufre una población infoxicada por los medios de comunicación social y las industrias culturales (Hesmondhalgh \& Baker, 2013). 
Por último, el desarrollo de proyectos transmedia como los aquí analizados son una oportunidad para la conformación de subjetividades políticas post-capitalistas (Alemán, 2014) de nuestros estudiantes por las que restablecer aquellas facultades humanas que han sido a nuestro juicio erosionadas en el actual universo cultural y mediático (Alba Rico, 2013), como son las de ejercer una racionalidad situada a través de prácticas narrativas que recuperan el sentido de historicidad presente en nuestra memoria y del bagaje mediático y cultural de afectos, necesidades, tecnologías, contextos y personas, todo ello en la búsqueda de una imaginación que proyecta sueños y horizontes en el ámbito de lo social y comunitario (Sobers, Dovey, Agusita, Baidoo, West, \& Chapain, 2014).

Como conclusión final, pensamos que las narrativas transmedia nos hablan de la necesidad de recuperar una perspectiva ecológica y multicultural del conocimiento (Sousa Santos, 2005; Irigaray \& Lovato, 2014) en la que se ponen a dialogar entre sí conocimientos expertos y conocimientos profanos (ecología de saberes), relacionando formas contemporáneas y no-contemporáneas presentes en la experiencia y memoria cultural de nuestros jóvenes y sus vínculos intergeneracionales (ecología de las temporalidades), recuperando formas de autogestión y cooperación para la realización de proyectos alejados de la ortodoxia de las industrias culturales (ecología de las productividades) y nos permiten comprender que nuestra experiencia tecnocultural se conforma al calor de contextos locales, regionales, estatales y globales (ecología de la trans-escala).

\section{Referencias bibliográficas}

Alba Rico, S. (2013). ¿Podemos seguir siendo de izquierdas? (Panfleto en sí menor). Barcelona: Pollen Ediciones

Alemán, J. (2014). En la frontera. Sujeto y capitalismo. Buenos Aires: Gedisa.

Allmer, T. (2015). Critical Theory and Social Media: Between Emancipation and Commodification (Vol. 144). New York: Routledge.

Ardévol, E, Estalella, A., \& Domínguez, D. (2008). La mediación tecnológica en la práctica etnográfica. Donostia: Anjulegi Atropolfia Elkartea.

Atkinson, S. (2014). The performative functions of dramatic communities: conceptualizing audience engagement in transmedia fiction. International Journal of Communication, 8, 2201-2219.

Bar, F., Weber, M. S., \& Pisani, F. (2016). Mobile technology appropriation in a distant mirror: Baroquization, creolization, and cannibalism. New media \& society, 18 (4).

Barbero, J. M. (1987). De los medios a las mediaciones. Barcelona: Gustavo Gili.

Beaulieu, A. (2004). Mediating ethnography: objectivity and the making of ethnographies of the internet. Social Epistemology, 18(2-3): 139-164.

Berry, D. M. (2015). Critical theory and the digital. USA: Bloomsbury Publishing

Booth, P. (2010). Digital fandom2.0: New media studies. US: Peter Lang.

Buckingham, D., \& Kehily, M. J. (2014). Introduction: Rethinking Youth Cultures in the Age of Global Media. In S. Bragg, M. J. Kehily \& D. Buckingham (Ed.). Youth Cultures in the Age of Global Media (pp. 1-18). UK: Palgrave Macmillan.

Castells, M. (2006). La sociedad red: una visión global. Madrid: Alianza.

Chase, S. E. (2011). Narrative Inquiry: Still a Field in the Making. In N.K. Denzin, \& Y. S. Lincoln (Ed). The SAGE Handbook of Qualitative Research. Los Angeles: SAGE Publications.

Cobo, C., \& Moravec, J. W. (2011). Aprendizaje Invisible. Hacia una nueva ecología de la Educación. Barcelona: Col.lecció Transmedia XXI. Laboratori de Mitjans Interactius / Publicacions I Edicions de la Universitat de Barcelona.

Cope, B., \& Kalantzis, M. (2009). Ubiquitous Learning. Exploring the anywhere/any time possibilities for learning in the age of digital media. Illinois, University of Illinois Press.

Dahlgren, P. (2013). The Political Web: Media, Participation and Alternative Democracy. New York: Palgrave Macmillan.

Davis A., Fidler, D., \& Gorbis. D. (2011). Future Work Skills 2020. Palo Alto, CA: Institute for the 
Future for University of Phoenix Research Institute.

Dede, C. (2010). Comparing frameworks for 21st century skills. In J. Bellanca \& R. Brandt (Eds.), 21 st century skills: Rethinking how students learn (pp. 51-76). Bloomington, IN: Solution Tree.

Denzin, N. K., \& Lincoln, Y. S. (2011). The SAGE Handbook of Qualitative Research. Los Angeles, SAGE Publications.

Fleming, L. (2013). Expanding learning opportunities with transmedia practices: Inanimate Alice as an exemplar. Journal of Media Literacy Education, 5(2), 3.

Fueyo, A.; De Andrés, S. (2017). Educación mediática: un enfoque feminista para deconstruir la violencia simbólica de los medios. Revista Fuentes, 19(2), 81-93.

Garnham, N. (2000). Emancipation, the media, and modernity: arguments about the media and social theory. OUP: Oxford.

Gea, M., Alaman, X., \& Rodriguez, P. (2016). Transmedia Storytelling for Social Integration of Children with Cognitive Disabilities. In V. Uskov, R. J. Howlett \& L. C. Jain (Eds.). Smart Education and e-Learning (pp. 463-471). Springer International Publishing.

Georgi, C., \& Glaser, B. J. (2016). Convergence Culture Reconsidered-Media-Participation-Environments. Universitätsverlag Göttingen.

Gibbs, G. (2012). El análisis de datos cualitativos en Investigación cualitativa. Madrid. Morata.

Gil de Zúñiga, H., Jung, N., \& Valenzuela, S. (2012). Social media use for news and individuals' social capital, civic engagement and political participation. Journal of Computer-Mediated Communication, 17(3), 319-336.

Hall, S. (1982). The rediscovery of ideology: Return of the repressed in media studies. Cultural theory and popular culture: $A$ reader, 111-141.

Hall, S. (2001). Encoding/decoding. Media and cultural studies: Keyworks, 166-176.

Hepp, A., \& Krotz, F. (Eds.). (2014). Mediatized worlds: Culture and society in a media age. UK: Palgrave Macmillan.

Hesmondhalgh, D., \& Baker, S. (2013). Creative labour: Media work in three cultural industries. London/New York: Routledge.

Hess, C. \& Ostrom, E. (eds.) (2016). Los bienes comunes del conocimiento. Madrid: Traficantes de Sueños. Hine, C. (2004). Etnografía virtual. Barcelona: UOC.

Irigaray, F., \& Lovato, A. (2014). Hacia una comunicación transmedia. Rosario: UNR.

Ito, M. (2010). Hanging Out, Messing Around, and Geeking Out. Massachusetts: MIT

Jenkins.H. (2003). Transmedia storytelling: Moving characters from books to films to video games can make them stronger and more compelling. MIT Technology Review, January 15.

Jenkins, H. (2006). Convergence Culture; Where Old and New Media Collide. New York: New York University Press

Jenkins, H., Ford, S., \& Green, J. (2013). Spreadable media: Creating value and meaning in a networked culture. New York: New York University Press.

Jenkins, H., Ito, M., \& Boyd, D. (2015). Participatory Culture in a Networked Era: A conversation on Youth, Learning, Commerce, and Politics. Cambridge, UK: Polity Press.

Kalogeras, S. (2014). Transmedia Storytelling and the New Era of Media Convergence in Higher Education. UK: Palgrave Macmillan.

Livingstone, S. (2012). Critical reflections on the benefits of ICT in education. Oxford review of education, 38(1), 9-24.

Malone, T. W., \& Bernstein, M. S. (2015). Handbook of Collective Intelligence. Cambridge, MA: MIT Press

Moravec, J. W. (2013). Knowmad society: the 'new' work and education. On the Horizon. Vol $21 \mathrm{n}^{\circ} 2$ 79-83

Rao, M. B., Jongerden, J., Lemmens, P., \& Ruivenkamp, G. (2015). Technological mediation and power: Postphenomenology, critical theory, and autonomist marxism. Philosophy \& Technology, 28(3), 449-474.

Rivas Flores, J. I. (2014). Narración frente al neoliberalismo en la formación docente. Visibilizar para transformar. Magis. Revista Internacional de Investigación en Educación 7 (14) 99-112 
Rogoff, B. (1997). Los tres planos de la actividad sociocultural: apropiación participativa, participación guiada y aprendizaje. En J. Wertsch, P. del Río, \& A. Álvarez (Eds.). La mente sociocultural. Aproximaciones teóricas y aplicadas (pp. 111-128). Madrid: Fundación Infancia y Aprendizaje.

Schrøder, K. C. (2012). Discursive Realities. In K. B. Jensen (Ed.), A Handbook of Media and Communication Research: Qualitative and Quantitative Methodologies. (pp. 106-130). London: Routledge.

Scolari, C.A. (2013). Narrativas Transmedia Cuando todos los medios cuentan. Barcelona, Deusto S.A. Ediciones.

Scolari, C. (2016). Alfabetismo transmedia. Estrategias de aprendizaje informal y competencias mediáticas en la nueva ecología de la comunicación. Telos, 103,13-23.

Scolari, C. (2018). Alfabetismo transmedia en la nueva ecología de los medios. Libro blanco. Consultado en: https://es.scribd.com/document/375759215/Scolari-Transmedia-Literacy-pdf(25/04/2018)

Scolari, C., Bertetti, P., \& Freeman, M. (2014). Transmedia archaeology: storytelling in the borderlines of science fiction, comics and pu巾 magarines. UK: Palgrave Macmillan.

Simons, H. (2009). El estudio de caso: teoría y práctica. Madrid: Morata.

Simons, N. (2011). Television audience research in the age of convergence: challenges and difficulties. In Proceddings of the 9th international interactive conference on Interactive television pp. 101-104. ACM.

Sobers, S., Dovey, J., Agusita, E., Baidoo, V., West, T., \& Chapain, C. (2014). Creative citizens: Researching the value of creative citizenship through co-creative action research (A 'transmedia' case study). In Creative Citizens: The Conference, Royal College of Art, London, UK, 18-19 September 2014

Soriano, C. R. R. (2016). Transmedia mobilization: agency and literacy in minority productions in the age of spreadable media. The Information Society, 32(5), 354-363.

Sousa Santos, B. (2005). El milenio buérfano: ensayos para una nueva cultura politica. Madrid: Trotta.

Stein, L. E. (2015). Millennial fandom: Television audiences in the transmedia age. University of Iowa Press.

Strauss, A., \& Corbin, J. (1990). Basics of qualitative research. P edición. Thousand Oaks: Sage.

Thompson, J. B. (1991). Ideology and modern culture: Critical social theory in the era of mass communication. Stanford University Press: John Wiley \& Sons.

Trinidad, A., Carrero, V. Soriano, R. M. (2006). Teoría fundamentada «Grounded Theory». La construcción de la teoría a través del análisis interpretacional. Madrid: Centro de Investigaciones Sociológicas.

Tubau, D. (2011). El guión del siglo 21: el futuro de la narrativa en el mundo digital. Madrid: Alba

Van Dijck, J. (2013). The Culture of Connectivity: A Critical History of Social Media. New York: Oxford University Press

Wängqvist, M., Frisén, A. (2016). Who am I On Line? Understanding the meaning of On Line Contexts for Identity Development. Adolescent Research Review, Volumen 1, Issue 2, 139-152.

Vélez, I. (2017). La formación en alfabetización mediática y competencia digital. Revista Fuentes, 19(2), 15-19.

Wood, H. (2014). Active Audience and Uses. In M. Alvarado, M. Buonanno, H. Gray \& T. Miller (Eds). The SAGE Handbook of Television Studies (p. 366). London: SAGE Publications.

\section{Para citar este artículo}

Anguita Martínez, R., De la Iglesia Atienza, L. \& García Zamora, E. (2018). Creación de contenidos transmedia en la sociedad hiperconectada. Una etnografía digital con jóvenes universitarios. Revista Fuentes, 20(1), 29-41. [Fecha de consulta: dd/mm/aa]. doi: http://dx.doi.org/10.12795/revistafuentes.2018.v20.11.02 